\title{
NO signalling in mice lacking the NO receptor guanylyl cyclase Dieter Groneberg${ }^{1}$, Peter König², Doris Koesling1 and Andreas Friebe*1
}

Address: ${ }^{1}$ Institut für Pharmakologie und Toxikologie, Medizinische Fakultät, Ruhr-Universität Bochum, Bochum, Germany and ${ }^{2}$ Institut für Anatomie, Medizinische Fakultät, Universität Lübeck, Lübeck, Germany

Email: Andreas Friebe* - andreas.friebe@rub.de

* Corresponding author

from 4th International Conference of cGMP Generators, Effectors and Therapeutic Implications

Regensburg, Germany. 19-21 June 2009

Published: II August 2009

BMC Pharmacology 2009, 9(SuppI I):SI2 doi:I0.1/86/I47I-22I0-9-SI-SI2

This abstract is available from: http://www.biomedcentral.com/I47I-22I0/9/SI/SI2

(c) 2009 Groneberg et al; licensee BioMed Central Ltd.

NO-sensitive guanylyl cyclase (NO-GC) is accepted to be the major receptor for the signaling molecule NO. NO-GC catalyzes the production of the intracellular second messenger CGMP and thereby has a key regulatory function regarding various physiological processes. NO-GC is made up of one $\beta$ subunit and one $\alpha$ subunit. On the protein level, there are two $\alpha$ subunits $\left(\alpha_{1}\right.$ and $\left.\alpha_{2}\right)$ and one $\beta$ subunit $\left(\beta_{1}\right)$ known, and thus two different GC isoforms exist $\left(\alpha_{1} \beta_{1}\right.$ and $\left.\alpha_{2} \beta_{1}\right)$.

In the enteric nervous system, non-adrenergic non-cholinergic (NANC) neurons control the motor function of the gastrointestinal tract. These NANC neurons express neuronal NO synthase. NO has been demonstrated to be an important physiological mediator of NANC relaxation of gastrointestinal smooth muscle, leading to relaxation via activation of NO-GC.

In this study, we intended to clarify the role of NO-GC with regards to gastrointestinal (GI) motility. Immunohistochemical analysis revealed that GI smooth muscle expresses only minor amounts of NO-GC. Interestingly, strong expression was detected in the interstitial cells of Cajal (ICC). Using mice in which exon 10 of the $\beta_{1}$ subunit is surrounded by loxP sites (floxed mice), we have generated mice lines that lack NO-GC either ubiquitously or specifically in smooth muscle. In isometric force studies we used endogenous NO production induced by electric field stimulation and pharmacological NO release using NO donors. Total gut transit time was measured to study the consequences of NO-GC deletion in vivo.
General lack of NO-GC abolishes NO-dependent relaxation of GI smooth muscle. Neither physiological concentrations of NO using NO donors nor electric field stimulation at low frequencies led to relaxation of gastric fundus strips or sphincters. The whole gut transit time was increased approximately three times compared with WT. This dysfunction led to early mortality starting around day 18 after birth. Mortality was reduced by omission of dietary fiber.

Surprisingly, in smooth muscle-specific knockout mice (SMKO) the NO-dependent relaxation was reduced, but still intact. Total gut transit time showed no difference and survival of SMKO animals was similar to that of WT mice. In conclusion, the NO receptor guanylyl cyclase in GI smooth muscle is dispensable for motility and survival. 